\title{
The Subgraph Isomorphism Problem on a Class of Hyperedge Replacement Languages
}

\author{
H.N. de Ridder ${ }^{1, \star}$ and N. de Ridder ${ }^{2, \star \star}$ \\ 1 University of Konstanz, Department of Computer and Information Science, \\ 78457 Konstanz, Germany \\ ernst.de-ridder@uni-konstanz.de \\ 2 Department of Computer Science, University of Rostock, 18051 Rostock, Germany
}

\begin{abstract}
A graph class is called $A$-free if every graph in the class has no graph in the set $A$ as an induced subgraph. Such characterisations by forbidden induced subgraphs are (among other purposes) very useful for determining whether $A$-free is a subclass of $B$-free, by determining whether every graph in $B$ has some graph in $A$ as an induced subgraph. This requires solving the Subgraph Isomorphism Problem, which is NP-complete in general, but for which effective practical algorithms for general and specific purposes exist. However, if $B$ is infinite, these algorithms cannot be used. We introduce Head-Mid-Tail grammars (a special case of hyperedge replacement grammars) which have the property that if an infinite set $B$ can be defined by a Head-Mid-Tail grammar then it is decidable whether every graph in $B$ contains some graph from a finite set $A$ of graphs as an induced subgraph, thereby solving the $A$-free $\subseteq B$-free problem. Moreover, our algorithm is both simple and efficient enough to be practical.
\end{abstract}

\section{Introduction}

\subsection{Notation}

In this article all graphs are simple and undirected, with edges written as unordered pairs $(u, v)$ of vertices. Let $G=(V, E)$ be a graph and $S \subseteq V$. The subgraph induced by $S$ is $G[S]=(S,\{(u, v) \mid(u, v) \in E \wedge u, v \in S\})$. Two graphs $G=(V, E)$ and $H=(W, F)$ are isomorphic if a bijection $\pi: V \rightarrow W$ exists, such that $(v, w) \in E$ iff $(\pi(v), \pi(w)) \in F$. A graph $G^{\prime}$ is an induced subgraph of $G$, written $G^{\prime} \sqsubseteq G$, iff there is some $S \subseteq V$ such that $G^{\prime}$ is isomorphic to $G[S]$. The complement $\bar{G}$ of $G$ has precisely those edges that are not in $G$ : $\bar{G}=(V, V \times V \backslash E)$ and the complement co- $\mathscr{C}$ of a graph class $\mathscr{C}$ consists of the complements of the graphs in $\mathscr{C}: \mathscr{C}=\{\bar{G} \mid G \in \mathscr{C}\}$. A graph class $\mathscr{C}$ is inducedhereditary if for every graph $G$ in $\mathscr{C}$, every induced subgraph of $G$ belongs to $\mathscr{C}$. For such classes a unique set $A$ of graphs exists, such that no graph $A^{\prime} \in A$ is in $\mathscr{C}$, but every proper induced subgraph of $A^{\prime}$ is. $\mathscr{C}$ then equals the class $A$-free of graphs that have no induced subgraph from the set $A$.

\footnotetext{
${ }^{\star}$ H.N. de Ridder: Part of the work done at the Department of Computer Science, University of Rostock, D-18051 Rostock, Germany.

${ }^{\star \star}$ N. de Ridder: Supported by Landesgraduiertenförderung Mecklenburg-Vorpommern, Germany.
} 


\subsection{Background}

Such forbidden subgraph characterisations have many uses:

1. They provide insight into the structure of graphs in the class.

2. Since a characterisation by minimal forbidden induced subgraphs is unique, it can be used for easily proving relations between graph classes.

3. They can be extremely helpful in the design of algorithms for problems like Independent Set or Colourability.

4. If a recognition algorithm for a class decides that the input does not belong to the class, it can return a forbidden subgraph in the input as an easily checkable certificate for the validity of the decision.

5. If the set of forbidden subgraphs is finite, then the graph class can be recognised in polynomial time by brute force comparison of all induced subgraphs of the input graph against the forbidden subgraphs.

For these reasons, finding forbidden subgraph characterisations is one of the evergreen topics in graph theory, with results ranging from König's classical theorem [12] that the bipartite graphs are precisely the odd cycle-free graphs, to the long open Strong Perfect Graph Theorem (SPGT ${ }^{1}$ ) [2] that the perfect graphs are precisely the (odd hole, odd anti-hole)-free graphs, where a hole is a chordless cycle with at least five vertices, an anti-hole the complement of a hole, an odd (anti-)hole is an (anti-)hole with an odd number of vertices.

It is the second aspect of forbidden subgraph characterisations, the determination of relations between graph classes, that interests us here in particular. Over the years, the mathematics and computer science communities have described many special graph classes in an effort to enlarge both our understanding of fundamental properties of graphs, and our ability to solve practical problems efficiently. This work has been and still is - so fruitful that very many classes have been defined, whose relations are difficult to overview even for the initiated. In an effort to make this field more accessible the book [1] documents over 300 classes. Out of this book sprang the online database ISGCI, the Information System on Graph Classes and their Inclusions [7]. The first version of ISGCI was released in 1999 with 300 graph classes and 10,000 inclusions and over the years it has grown to its current contents of about 1500 classes and over 170,000 inclusions, plus other relations. It provides the user with the ability to

- find the definition of graph classes;

- check the relation between graph classes and get a witness for these relations;

- find common super/subclasses of given graph classes;

- find the complexity of selected algorithmic problems on specific graph classes;

- find graph classes which are open with respect to the complexity of selected algorithmic problems;

- print inclusion diagrams of graph classes, optionally coloured according to the complexity of a specific problem;

- and provide literature references on graph classes, inclusions and algorithms. 


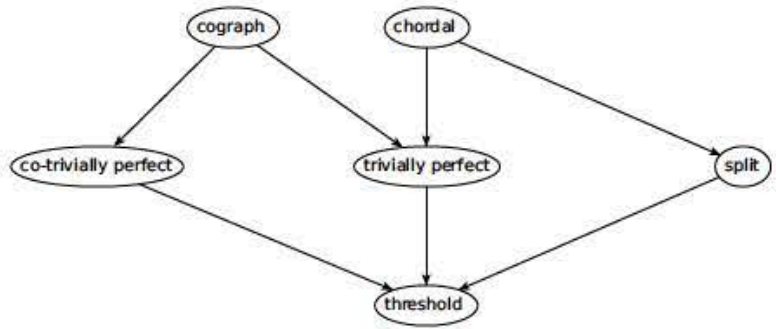

Fig. 1. Inclusion relations between some famous graph classes. There is an arrow $A \rightarrow B$ if $A \supseteq B$ is known.

Currently, the focus of development on ISGCI is on the user interface, wikification and graph parameters (cliquewidth etc.).

For a system like ISGCI completeness and soundness are two major goals. This not only refers to getting classes into the system, but especially to relations between classes that are in the system. For any two classes $\mathscr{A}, \mathscr{B}$ in the database the objective is to know as precisely as possible the relation between them: $\mathscr{A}=\mathscr{B}, \mathscr{A} \subseteq \mathscr{B}, \mathscr{A} \subsetneq \mathscr{B}$, $\mathscr{A} \cap \mathscr{B}=\emptyset$, or $\mathscr{A}, \mathscr{B}$ are incomparable (i.e. $\mathscr{A} \cap \mathscr{B}, \mathscr{A}-\mathscr{B}, \mathscr{B}-\mathscr{A}$ are all non-empty). To this end ISGCI incorporates a rule engine that derives new relations and checks them. As a simple example, if graph classes split, cograph, cograph $\cap$ split are present in the database, then from the rule $\mathscr{C}_{1} \cap \mathscr{C}_{2} \cap \ldots \mathscr{C}_{n} \subseteq \mathscr{C}_{i}$ we can deduce without further knowledge about the definition of split and cograph that cograph $\cap$ split $\subseteq$ split. But from this data we cannot deduce whether the inclusion is proper. Take a look at Fig. 1. It shows 6 classes $^{2}$ with (taking transitivity into account) 9 out of 15 relations.

All of these classes have in fact known characterisations by forbidden subgraphs, see Fig. 3 and Fig. 2, and from the forbidden subgraphs we can derive much more information.
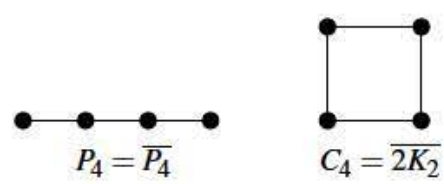

$C_{4}=\overline{2 K_{2}}$

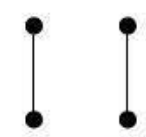

$2 K_{2}=\overline{C_{4}}$

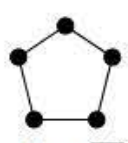

$C_{5}=\overline{C_{5}}$

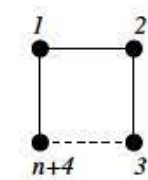

$C_{n+4}(n \geq 0)$

Fig. 2. The forbidden subgraphs for the classes in Fig. 3

\footnotetext{
${ }^{1}$ Formerly known as SPGC - Strong Perfect Graph Conjecture.

${ }^{2}$ These classes are well-known and well-studied. In the discussion that follows we aim to demonstrate the power of forbidden subgraph characterisations. Many of the relations we derive are also obtained in the literature using structural characterisations of these graph classes. This is a consequence of how well-studied these classes are and does not detract from the power and generality of the derivations sketched.
} 


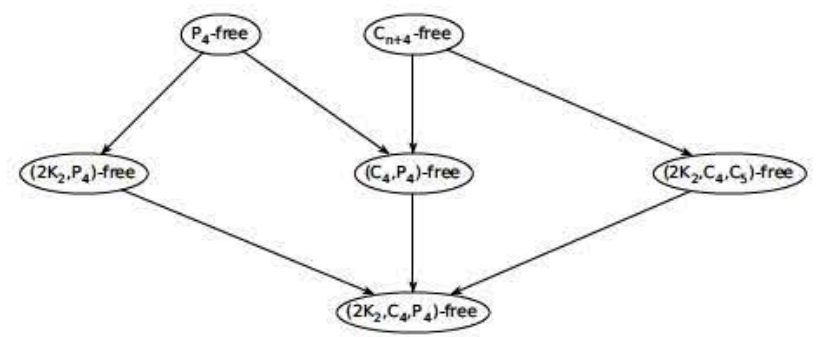

Fig. 3. Inclusion relations between the graph classes from Fig. 1, now characterised by forbidden subgraphs depictured in Fig. 2. Every class is drawn in the same position as in Fig. 1.

Definition 1. Let $A$ and $B$ be sets of graphs. $A$ forbids $B$ iff for every graph $B^{\prime} \in B$ there is a graph $A^{\prime} \in A$ such that $A^{\prime} \sqsubseteq B^{\prime}$.

So $A$-free $\subseteq B$-free iff $A$ forbids $B$. For convenience, we use the same term for single graphs: $A$ forbids $B$ iff $A$ is an induced subgraph of $B$.

Applying this together with some basic set theory to our example we arrive at the following conclusions:

- First of all, the forbidden subgraphs allow us to derive $\subseteq$-relations, e.g. threshold $\subseteq$ split follows from $\left\{2 K_{2}, C_{4}, P_{4}\right\}$ forbidding $\left\{2 K_{2}, C_{4}, C_{5}\right\}$.

- Then, because $\left\{2 K_{2}, C_{4}, C_{5}\right\}$ does not forbid $\left\{2 K_{2}, C_{4}, P_{4}\right\}$ (none of $2 K_{2}, C_{4}, C_{5}$ forbids $P_{4}$ ), it follows that split $\nsubseteq$ threshold and therefore that the inclusion is proper: threshold $\subsetneq$ split.

- For the properness of this relation we also get a witness: $P_{4}$ is a split graph but not a threshold graph.

- The forbidden subgraphs of the intersection of cograph and split are $2 K_{2}, C_{4}, P_{4}, C_{5}$. Because $P_{4} \sqsubseteq C_{5}$, we get cograph $\cap$ split $=\left(2 K_{2}, C_{4}, P_{4}\right)$-free, which are the threshold graphs. Hence, $\operatorname{cograph} \cap$ split $=$ threshold.

- The complement of a $\left(2 K_{2}, C_{4}, P_{4}\right)$-free graph is $\left(\overline{2 K_{2}}, \overline{C_{4}}, \overline{P_{4}}\right)$-free, which is to say $\left(C_{4}, 2 K_{2}, P_{4}\right)$-free. Hence threshold $=$ co-threshold, or threshold is self-complementary. Note that this does not imply that every threshold graph is isomorphic to its complement, but rather that every threshold graph is the complement of some threshold graph. Similar statements apply to cographs and split graphs.

- If we take any two classes in the diagram that have no inclusion relation drawn between them (possibly over multiple edges), then we can verify by the forbidden subgraphs that there indeed is no inclusion between them. For example, cograph $\nsubseteq$ split and split $\not \subset$ cograph with witnesses $P_{4}$ and $2 K_{2}$, respectively. Our diagram is therefore complete: every inclusion relation is indeed depicted.

Thus, using forbidden subgraphs improved our knowledge from $9 \subseteq$-relations and 6 unknowns to $9 \subset$-relations, 6 incomparables and 0 unknowns. Moreover, we have witnesses for everything. As a further indication of the importance of forbidden subgraphs for deducing relations, in ISGCI more than half of the inclusions alone (so not counting incomparabilities) are due to forbidden subgraph characterisations. 
The uses of forbidden subgraphs don't even end here. Because not only does $A$ forbid $B$ imply $A$-free $\subseteq B$-free, but also $A$-free $\subseteq B$-free implies that $A$ forbids $B$, these characterisations are of great importance to the verification of the soundness of the system.

- If the inclusion $A$-free $\subseteq B$-free is present in the database, then it must be that $A$ forbids $B$, or there is an error (either in the input or in the derivation).

\subsection{Goal}

All of this hinges on being able to determine by computer program whether a set of graphs $A$ forbids a set of graphs $B$ and, if not, giving a witness.

If $A$ and $B$ are both single graphs, then this asks whether $A \sqsubseteq B$, which is known as the Subgraph Isomorphism Problem. When $A \nsubseteq B$, the witness is $B$ itself. The Subgraph Isomorphism Problem is a generalisation of the Hamiltonian Cycle and Maximum Clique problems and is NP-complete in general [10]. As it has applications in pattern recognition, computer vision, biocomputing and databases, among others, practical solutions are in much demand. The classical algorithm for the general case is due to Ullman [15], and $[9,13]$ are surveys comparing the performance of current state of the art algorithms.

If $A$ and $B$ are finite sets, then we can loop through them according to Def. 1 and solve the Subgraph Isomorphism Problem for each pair $A^{\prime}, B^{\prime}$. In fact, this is precisely what ISGCI does. If we find some $B^{\prime}$ that is not forbidden by $A$, then this $B^{\prime}$ is our witness.

However, when (one of) these sets is infinite, this doesn't work anymore. And infinite sets of forbidden subgraphs are not at all rare. A set of graphs with a common structure that is used in a forbidden subgraph characterisation is called a family. Infinite families we have seen are the odd cycles for bipartite graphs; odd holes and odd anti-holes for perfect graphs; and $C_{n+4}$ for chordal graphs ${ }^{3}$.

In this article we let $B$ be infinite and study the problem of deciding whether every member of $B$ contains an induced subgraph in the finite set $A$. We shall have more to say on the case of infinite $A$ in Sec. 3. We introduce Head-Mid-Tail grammars, or HMTgrammars for short, which are a special case of hyperedge replacement grammars [11] and use these to solve the following specialization of the "forbidding" problem:

Problem 2. Given a finite set of graphs $A$ and a Head-Mid-Tail grammar $\mathscr{G}$, does $A$ forbid the graphs generated by $\mathscr{G}$ ?

In case of a negative answer, a good solution should also provide a witness.

It is important that $A$ is a set and not just a single graph, because often multiple graphs are needed to forbid a family. For example, in Sec. 1.2, the $\left(C_{4}, P_{4}\right)$-free graphs are a subclass of $C_{n+4}$-free graphs, because $C_{4}$ forbids $C_{4}$ and $P_{4}$ forbids $C_{5}$ and larger cycles.

We show that to solve Problem 2 it suffices to examine only a finite subset of the graphs generated by $\mathscr{G}$ and give an efficient procedure to calculate this subset. This results in an algorithm that is simple and efficient enough to be practical and can also return a witness when $A$ does not forbid the graphs of $\mathscr{G}$.

${ }^{3}$ Although these families are all cycles of some constrained length, or complements thereof, many families with another structure exist, as well. 


\section{Head-Mid-Tail Grammars}

\subsection{Definition}

Definition 3. The graphs we consider in this paper are tuples $X=\left(V_{X}, E_{X}\right.$, att $_{X}$, ext $\left._{X}\right)$, with $V_{X}($ or $V(X))$ and $E_{X}($ or $E(X))$ the vertex and edge sets of $X$, respectively, and att $_{X}$, ext $_{X}$ are bijective functions numbering a subset of vertices, with domains $\underline{a t t}_{X}$ and $\underline{\text { ext }}_{X}$, respectively:

$$
\begin{aligned}
& a^{a t t_{X}}: \underline{\text { att }}_{X} \rightarrow\left[1 \ldots\left|\underline{a t t}_{X}\right|\right], \quad \underline{a t t}_{X} \subseteq V_{X} \\
& \operatorname{ext}_{X}: \underline{\text { ext }}_{X} \rightarrow\left[1 \ldots\left|\underline{e x t_{X}}\right|\right], \quad \underline{\text { ext }} \underline{x}_{X} \subseteq V_{X}
\end{aligned}
$$

For a set of vertices $S$, $\operatorname{att}_{X}(S)=\left\{\operatorname{att}_{X}(v) \mid v \in S\right\}$, where implicitly $S \subseteq \underline{a t t}_{X}$. Analogously for $\operatorname{ext}_{X}(S)$.

If a graph has no attachment (extension), then we consider its attachment (extension) empty. We will number the vertices in $\underline{a t t}_{X}$ with $1 a, 2 a, \ldots$ where $\operatorname{att}_{X}(1 a)=$ $1, \operatorname{att}_{X}(2 a)=2, \ldots$ and similarly the vertices in $\underline{e x t}_{Y}$ with $1 e, 2 e, \ldots$

We call an ordered pair $(X, Y)$ compatible if $\left|\underline{a t t}_{X}\right|=\left|\underline{e x t}_{Y}\right|$. Let $X, Y$ be disjoint graphs such that the pair $(X, Y)$ is compatible and let $k:=\left|\underline{a t t}_{X}\right|=\left|\underline{e x t}_{Y}\right|$. The graph $X Y$ (the juxtaposition of $X$ and $Y$ ) is the composition of $X$ and $Y$ and is defined as the union of $X$ and $Y$ such that vertices with the same number in $\underline{a t t_{X}}, \underline{\text { ext }}_{Y}$ get identified ( $1 a \in \underline{a t t}_{X}$ with $1 e \in \underline{e x t}_{Y}, 2 a \in \underline{a t t}_{X}$ with $2 e \in \underline{e x t}_{Y}$ and so on) and all multiple edges are made simple. The formal definition is as follows:

Definition 4. Let $X, Y$ be graphs such that the pair $(X, Y)$ is compatible.

- $\lambda_{X Y}: V(Y) \rightarrow V(X Y)$ is defined by

$$
\lambda_{X Y}(y)=\left\{\begin{array}{ll}
y & \text { if } y \notin \underline{\operatorname{ext}} \underline{\mathrm{ex}}_{Y} \\
\operatorname{att}_{X}^{-1}\left(\operatorname{ext}_{Y}(y)\right) & \text { if } y \in \underline{\operatorname{ext}} Y
\end{array} .\right.
$$

When there is no danger of confusion we shall leave out the subscript ${ }_{X Y}$ and write simply $\lambda$.

- Without loss of generality, assume $X, Y$ are disjoint (otherwise work with isomorphic copies of $X, Y)$. The composition $X Y$ is defined up to isomorphism by

$$
\begin{aligned}
& \circ V(X Y)=V(X) \cup V(Y) \backslash \underline{e x t}_{Y}, \\
& \circ E(X Y)=E(X) \cup\left\{\left(\lambda\left(y^{\prime}\right), \lambda\left(y^{\prime \prime}\right)\right) \mid\left(y^{\prime}, y^{\prime \prime}\right) \in E(Y)\right\}, \\
& \circ \operatorname{att}_{X Y}=\operatorname{att}_{Y} \circ \lambda^{-1}, \\
& \circ \operatorname{ext}_{X Y}=\operatorname{ext}_{X} .
\end{aligned}
$$

Note that composition never deletes vertices nor edges. According to this definition edge-preserving functions $\chi: V(X) \rightarrow V(X Y)$ and $v: V(Y) \rightarrow V(X Y)$ exist. To keep the notation light, we shall leave out these functions and write in $X Y$ simply $S$ instead of $\chi(S)$ or $v(S)$, for $S \subseteq V(X)$ and $S \subseteq V(Y)$, respectively. For the $i$-repeated composition of a graph $X$ with itself we write $X^{i}$, with the individual copies of $X$ numbered with a subscript: $X^{i}=X_{1} X_{2} \ldots X_{i}$. 
Proposition 5. Composition is associative: $X(Y Z)=(X Y) Z$.

Definition 6. A Head-Mid-Tail grammar $\mathscr{G}$ is a tuple $(H, M, T)$ where

- $H$ is a graph with att ${ }_{H} \neq \varnothing$ and ext $t_{H}=\varnothing$;

- $M$ is a graph with att $_{M} \neq \varnothing$ and ext $_{M} \neq \varnothing$;

- $T$ is a graph with att $_{T}=\varnothing$ and ext $\neq \varnothing$;

- $\left|\underline{\text { att }}_{H}\right|=\left|\underline{e x t}_{M}\right|=\left|\underline{\text { att }}_{M}\right|=\left|\underline{e x t}_{T}\right|>0$. That is, the pairs $(H, M),(H, T),(M, M)$, $(M, T)$ are compatible.

A Head-Mid-Tail grammar $\mathscr{G}=(H, M, T)$ is called growing if $\underline{e x t}_{M} \neq V(M)$. The language $L(\mathscr{G})$ generated by a given Head-Mid-Tail grammar $\mathscr{G}=(H, M, T)$ is the family of all graphs that can be composed from $H, i$ copies of $M(i \geq 0, i \in \mathbb{N})$ and $T$ :

$$
H T\left(=H M^{0} T\right), H M T\left(=H M^{1} T\right), H M M T\left(=H M^{2} T\right), \ldots
$$

Proposition 7. $L(\mathscr{G})$ is infinite iff $\mathscr{G}$ is growing.

Note that for every graph in $L(\mathscr{G})$ both attachment and extension are empty. As an example, Fig. 4 gives a Head-Mid-Tail grammar for the family holes. We remark that the holes, like many other families, cannot be generated by iterated composition of only two graphs.
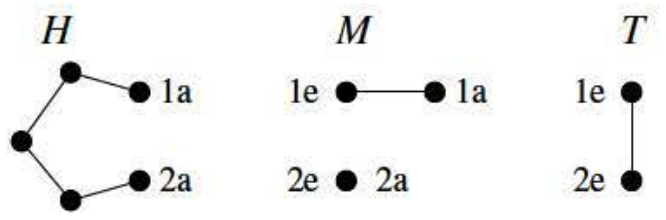

Fig. 4. $\mathscr{G}=(H, M, T)$ generating the family Holes

Head-Mid-Tail grammars are a special case of hyperedge replacement grammars, where att, ext and composition have their origin. We'll have more to say on hyperedge replacement grammars in Sec. 3.

\subsection{Normalised Head-Mid-Tail Grammars}

Infinite families of graphs that can be represented with a Head-Mid-Tail grammar may have several different such representations. Our algorithm for solving Problem 2 is applied to normalised Head-Mid-Tail grammars. Before explaining what this is we need some auxiliary definitions. 
Let $\mathscr{G}=(H, M, T)$ be a Head-Mid-Tail grammar. Define $Q(M):=\underline{e x t}_{M} \cap \underline{a t t}_{M}$ and $q:=|Q(M)|$. A transitive cycle of $M$ is a minimal set of vertices $C \subseteq Q(M)$ such that ext $_{M}(C)=\operatorname{att}_{M}(C)$. The transitive set of $M$ is the maximal set of vertices $M_{\mathrm{tr}} \subseteq Q(M)$ such that $\operatorname{ext}_{M}\left(M_{\mathrm{tr}}\right)=\operatorname{att}_{M}\left(M_{\mathrm{tr}}\right)$. We remark that $M_{\mathrm{tr}}$ is the maximal set of vertices on which $\lambda_{M M}$ is a permutation and that a transitive cycle is precisely an orbit of $\lambda_{M M}$. Clearly, $M_{\mathrm{tr}}=\bigcup_{C} C$ and $M_{\mathrm{tr}} \subseteq Q(M)$. See Fig. 5 for an example of transitive cycles and the transitive set of $M$.

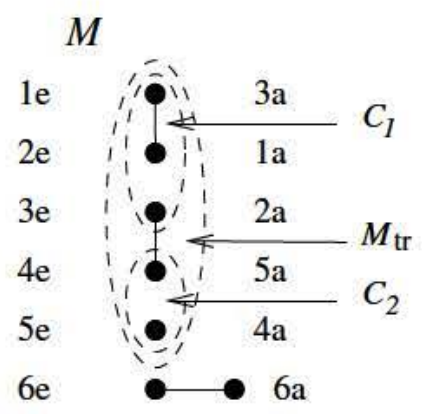

Fig. 5. Transitive cycles $C_{1}, C_{2}$, transitive set $M_{\mathrm{tr}}$. Here $Q(M)=M_{\mathrm{tr}}$.

If the pair $(X, M)$ is compatible, then we define $X_{\mathrm{tr}}:=\operatorname{att}_{X}^{-1}\left(\operatorname{ext}_{M}\left(M_{\mathrm{tr}}\right)\right)$ and if $(M, X)$ is compatible, then $X_{\mathrm{tr}}:=\operatorname{ext}_{X}^{-1}\left(a t t_{M}\left(M_{\mathrm{tr}}\right)\right)$. Well-definedness requires that if both $(M, X)$ and $(X, M)$ are compatible then these definitions must be equal and for $X=M$, the solution to this equality is given by the definition of $M_{\mathrm{tr}}$ above.

Definition 8. A Head-Mid-Tail grammar $\mathscr{G}=(H, M, T)$ is called normalised if

1) $Q(M)=M_{\mathrm{tr}}$;

2) $\forall v \in M_{\mathrm{tr}}: \operatorname{ext}_{M}(v)=\operatorname{att}_{M}(v)$;

3) $M\left[M_{\mathrm{tr}}\right]$ is edgeless.

Intuitively, in all graphs $H M^{i}$ generated by a normalised grammar, $H_{\text {tr }}$ induces the same subgraph: By 8.1 its set of vertices doesn't change; by 8.2 the attachment/extension numbering doesn't change; and by 8.3 its set of edges doesn't change. The consequences and use of being normalised will be established in section 2.3.

Every Head-Mid-Tail grammar can be reformulated as a set of normalised grammars.

Theorem 9. For any Head-Mid-Tail grammar $\mathscr{G}=(H, M, T)$ we can construct a finite set $S$ of graphs and a finite set of normalised grammars $\mathscr{G}_{i}^{\prime}$ such that $S \cup \bigcup_{i} L\left(\mathscr{G}_{i}^{\prime}\right)=L(\mathscr{G})$.

Proof. See [6]. 


\subsection{Properties of Normalised Grammars}

We next prove some properties of normalised grammars.

Lemma 10. Let $\mathscr{G}=(H, M, T)$ be normalised. Then in graphs $H M^{i} T, \forall i>0$ the sets $\underline{\text { att }}_{H} \backslash H_{\mathrm{tr}}, \underline{\text { ext }}_{T} \backslash T_{\mathrm{tr}}$ and $\underline{\text { ext }}_{M_{j}} \backslash M_{\mathrm{tr}}, \forall j, 2 \leq j \leq i$ are pairwise disjoint.

Proof. By definition of transitive set, in $H M^{i} T: \underline{\text { att }}_{H} \backslash H_{\mathrm{tr}}=\underline{\text { ext }}_{M_{1}} \backslash M_{\mathrm{tr}}$ and $\underline{e x t}_{T} \backslash T_{\mathrm{tr}}=$ $\underline{\text { att }}_{M_{i}} \backslash M_{\mathrm{tr}}$. If we define $\underline{\text { ext }}_{M_{i+1}}:=\underline{e x t}_{T}$, then $\forall j: 1 \leq j \leq i, \forall k: 1 \leq k \leq i-j+1$ $\underline{e x t}_{M_{j}} \backslash M_{\mathrm{tr}} \cap \underline{e x t}_{M_{j+k}} \backslash M_{\mathrm{tr}}=\left(\underline{e x t}_{M_{j}} \cap \underline{e x t}_{M_{j+k}}\right) \backslash M_{\mathrm{tr}} \subseteq\left(\underline{e x t}_{M_{j}} \cap \underline{\text { att }}_{M_{j}}\right) \backslash M_{\mathrm{tr}}$, because by definition of composition all vertices of $M_{j}$ that are in $M_{j+1} \ldots M_{i}$ are in $\underline{a t t}_{M_{j}}$. By definition of $Q(M)$, this equals $Q(M) \backslash M_{\mathrm{tr}}=\varnothing$, as by Def. 8.1, $Q(M)=M_{\mathrm{tr}}$.

Definition 11. Consider a normalised grammar $\mathscr{G}=(H, M, T)$. We say that a graph $X$ fits easily into $H M^{i} T$ if $X \sqsubseteq H M^{i} T$ such that at least one of the following conditions holds:

a) $V(X) \cap \underline{a t t}_{H} \backslash H_{\mathrm{tr}}=\varnothing$ or

b) $V(X) \cap \underline{e x t}_{T} \backslash T_{\text {tr }}=\varnothing$ or

c) $\exists j, 2 \leq j \leq i: V(X) \cap \underline{\operatorname{ext}}_{M_{j}} \backslash M_{\mathrm{tr}}=\varnothing$.

In particular, if $X$ fits easily into $H T$ then both a) and b) hold. See Fig. 6: if $X$ fits easily into $H M^{i} T$, then $X$ has no vertices in at least one of the hatched areas.

Theorem 12. Let $\mathscr{G}=(H, M, T)$ be growing and normalised and let $X$ be a graph. If $|V(X)| \leq i$, then $X \sqsubseteq H M^{i} T$ iff $X$ fits easily into $H M^{i} T$.

Proof. $\Longleftarrow:$ follows from Def. 11 .

$\Longrightarrow$ : Let $X \sqsubseteq H M^{i} T,|V(X)| \leq i$. Suppose $X$ does not fit easily into $H M^{i} T$. Then:

- $\left|V(X) \cap \underline{a t t}_{H} \backslash H_{\mathrm{tr}}\right| \geq 1$,

- $\left|V(X) \cap \underline{\text { ext }}_{T} \backslash T_{\mathrm{tr}}\right| \geq 1$,

- $\forall j, 2 \leq j \leq i:\left|V(X) \cap \underline{e x t}_{M_{j}} \backslash M_{\mathrm{tr}}\right| \geq 1$.

By Def. 8.1, Lemma 10 holds, thus $|V(X)| \geq i+1$ - contradiction.

Theorem 13. Let $\mathscr{G}=(H, M, T)$ be growing and normalised and let $X$ be a graph. Let $i$ be the smallest exponent such that $X$ fits easily into $H M^{i} T$, then $i \leq|V(X)|$.

Proof. Let $i$ be the smallest exponent such that $X$ fits easily into $H M^{i} T$. Assume $i>$ $|V(X)|$, then $X$ does not fit easily into $H M^{|V(X)|} T$. It follows that

- $\left|V(X) \cap \underline{a t t}_{H} \backslash H_{\mathrm{tr}}\right| \geq 1$,

- $\left|V(X) \cap \underline{\operatorname{ext}}_{T} \backslash T_{\mathrm{tr}}\right| \geq 1$,

- $\forall j, 2 \leq j \leq|V(X)|:\left|V(X) \cap \underline{e x t}_{M_{j}} \backslash M_{\mathrm{tr}}\right| \geq 1$.

By Def. 8.1, Lemma 10 holds, thus $|V(X)| \geq|V(X)|+1$ - contradiction. 


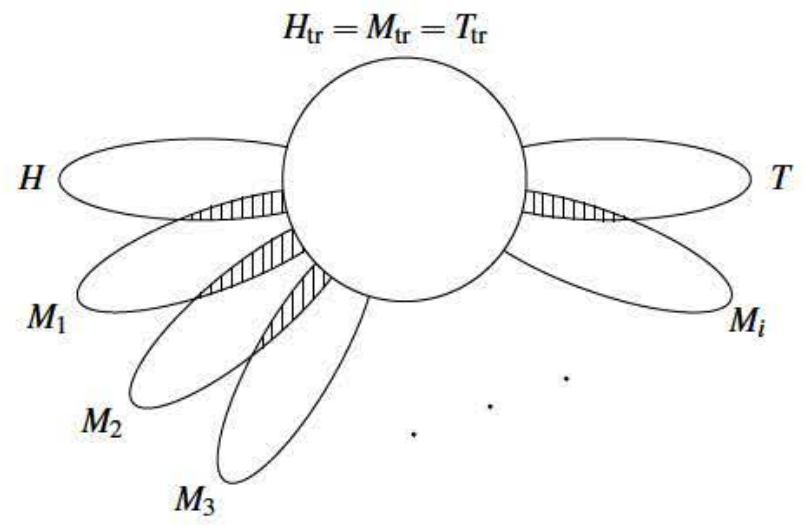

Fig. 6. $\mathscr{G}=(H, M, T)$ is normalised. Illustration for Def. 11 .

The next theorem formalizes the following intuition: If a graph $X$ fits easily into $H M^{i}$, then $X$ has no vertices in one of the disjoint hatched areas of Fig. 6 . As discussed after Def. 9 , the graph induced by $H_{\mathrm{tr}}$ doesn't change by pumping. Hence, we can insert new copies of $M$ precisely in a hatched area without changing any vertex of $X$. Thereby $X$ is also an induced subgraph of all $H M^{l} T$, with $l>i$.

Theorem 14. Let $\mathscr{G}=(H, M, T)$ be growing and normalised and let $X$ be a graph. If $X$ fits easily into $H M^{i} T$, then $X$ fits easily into $H M^{l} T$, for all $l>i$.

Proof. See [6].

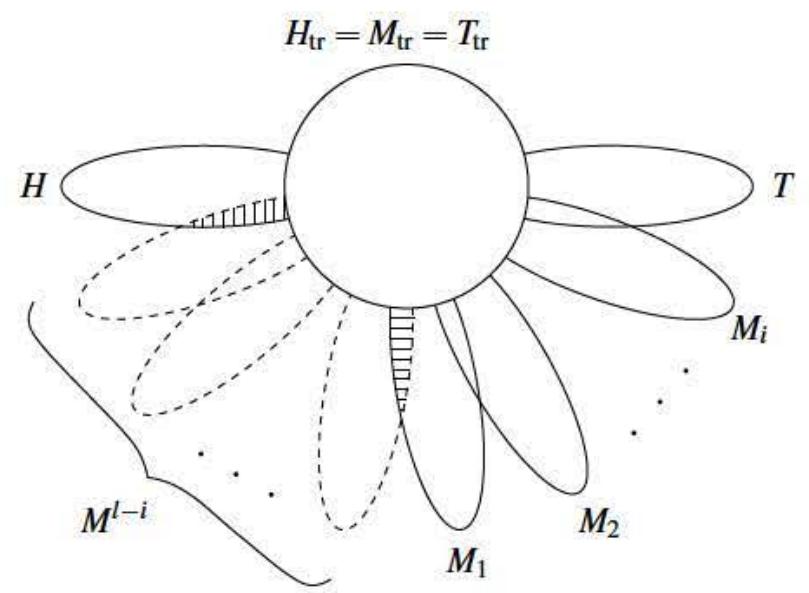

Fig. 7. $\mathscr{G}=(H, M, T)$ is normalised. Illustration for Th. 14 


\subsection{The Algorithm}

After this preparatory work on normalised grammars, we are going to exploit their properties algorithmically. The first theorem is conceptually part of Sec. 2.3, but since it is the basis of our algorithm, we list it here.

Theorem 15. Let $\mathscr{G}=(H, M, T)$ be growing and normalised, let $X$ be a graph and let $i=|V(X)|$. Then either $\forall l \geq i: X \sqsubseteq H M^{l} T$ or $\forall l \geq i: X \nsubseteq H M^{l} T$.

Proof. If $X$ fits easily into $H M^{i} T$, then, by Th. 14, $X$ fits easily into $H M^{l} T$ for all $l>i$, as well, and it follows that $\forall l \geq i: X \sqsubseteq H M^{l} T$.

If $X$ does not fit easily into $H M^{i} T$, then, by Th. $14, X$ does not fit easily into $H M^{k} T, \forall k \leq i$. By Th. 13, there is no $j$ such that $X$ fits easily into $H M^{j} T$. Thus, by Th. $12, \forall l \geq i: X \nsubseteq H M^{l} T$.

We remark that this theorem implies that (for a normalised grammar!) situations where, for example, $X$ forbids all the odd members of $L(\mathscr{G})$, but not the even members, and $Y$ forbids the even members, but not the odd members, are not possible. For general Head-Mid-Tail grammars such situations are possible. For $X$ and a normalised grammar, either the set of forbidden members or the set of non-forbidden members is finite and a subset of the $|V(X)|$ smallest members.

Theorem 16. Let $\mathscr{G}=(H, M, T)$ be growing and normalised and let $W$ be a finite set of graphs. W forbids $L(\mathscr{G})$ iff $W$ forbids $\left\{H M^{i} T\left|0 \leq i \leq \max _{X \in W}\right| V(X) \mid\right\}$.

Proof. $W$ forbids $L(\mathscr{G})$ means that $\forall i \geq 0 \quad \exists X \in W: X \sqsubseteq H M^{i} T$. Let $t:=\max _{X \in W}|V(X)|$. Then we can rewrite what we need to prove as follows:

$$
\forall i \geq 0 \exists X \in W: X \sqsubseteq H M^{i} T \quad \Longleftrightarrow \quad \forall i: 0 \leq i \leq t \exists X \in W: X \sqsubseteq H M^{i} T
$$

$\Longrightarrow$ : Obvious.

$\Longleftarrow$ : By assumption, $Y \in W$ exists such that $Y \sqsubseteq H M^{t} T$. By Th. 15 and definition of $t$, we get $\forall i \geq t: Y \sqsubseteq H M^{i} T$. Together with the assumption, the statement follows.

Algorithm 17 (Does a finite set of graphs forbid a normalised grammar?) The following algorithm decides for a finite set of graphs $W$ and a growing, normalised HeadMid-Tail grammar $\mathscr{G}=(H, M, T)$ whether $W$ forbids $L(\mathscr{G})$. 


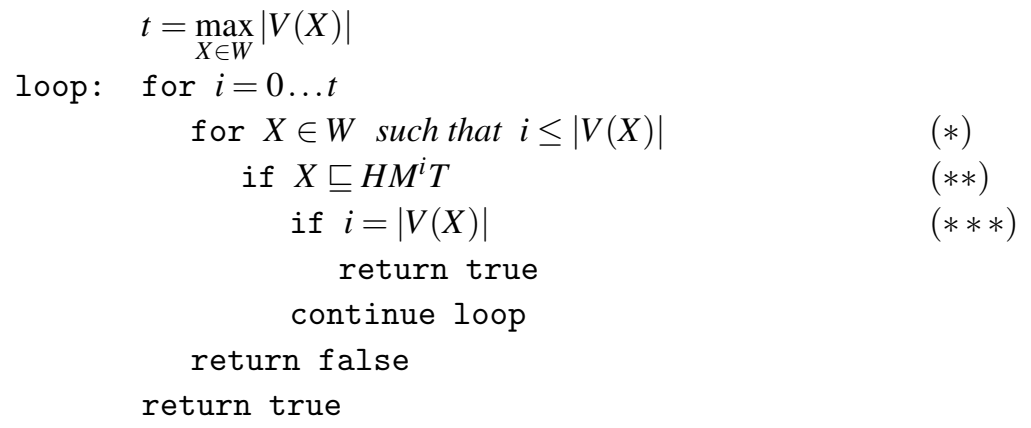

Proof. The algorithm is a straightforward implementation of Th. 16, with two optimizations: By Th. 15 we don’t need to check whether $X \sqsubseteq H M^{i} T$ for $i>|V(X)| \quad(*)$ and can return true immediately when $X \sqsubseteq H M^{|V(X)|} T \quad(* * *)$.

If at $(* *)$ a subgraph isomorphism algorithm is used that not only returns true/false, but also returns the actual subgraph, if one exists, then we can add a further optimization at $(* * *)$ by returning true as soon as either $i=|V(X)|$ or $X$ fits easily into $H M^{i} T$. If a witness is required, then we can return the witness $H M^{i} T$ together with the result false.

Theorem 18. There is a simple algorithm that for a finite set of graphs $W$ and a HeadMid-Tail grammar $\mathscr{G}$ decides whether $W$ forbids $L(\mathscr{G})$.

Proof. If $\mathscr{G}$ is not normalised, then by Th. 9 we can construct $S$ and $\mathscr{G}_{i}^{\prime}$ such that $S \cup \bigcup_{i} L\left(\mathscr{G}_{i}^{\prime}\right)=L(\mathscr{G})$, with $\mathscr{G}_{i}^{\prime}$ normalised. Check $S$. Check all $\mathscr{G}_{i}^{\prime}$ (Alg. 17).

By Th. 15, we can formulate similar algorithms to check whether $W$ forbids any graph in $L(\mathscr{G})$, or no graph in $L(\mathscr{G})$.

\section{Discussion}

By Alg. 17 we have that Problem 2 is at most as hard as the subgraph isomorphism problem, which is NP-complete [10]. In the other direction, by using a Head-Mid-Tail grammar that generates only a single graph, we can reduce the subgraph isomorphism problem to Problem 2. Hence Problem 2 is NP-complete, as well. Our algorithm makes a linear number of calls to a subgraph isomorphism algorithm and performance heavily depends on the subgraph algorithm used. Comparisons of different subgraph isomorphism algorithms are [9,13]; ISGCI uses VFLib [16] which implements the VF2 algorithm [4].

Although it is straightforward [6] to implement Th. 9 in order to normalise any given Head-Mid-Tail grammar, in practice it has turned out to be better to skip this step and instead require that grammars be presented normalised: Because normalised grammars are far easier to understand for humans than not-normalised ones, this reduces the number of input mistakes. 
Head-Mid-Tail grammars are a special case of hyperedge replacement grammars $[11,14]$ limited to producing simple graphs: Consider a hyperedge replacement grammar $G=(N, T, P, S)$, such that $N=\{S, A\}$ and $P=\{S:=$ Head, $A:=$ Mid $\mid$ Tail $\}$, where Head is an $A$-handled hypergraph, Mid is an $A$-handled type(A)-hypergraph and Tail is a type $(A)$-hypergraph, then we can define $G$ by the tuple (Head, Mid, Tail). The theory of hyperedge replacement grammars has a mechanism for deciding whether every graph generated by a grammar satisfies a certain property, if that property is so-called compatible. Roughly speaking, a compatible property can be decided for a graph by combining the property (or a related one) on the components used in the derivation of the graph. Unfortunately, the induced subgraph property is not compatible and therefore this mechanism doesn't help us in solving Problem 2. Another approach is expressing the subgraphs in monadic second order logic, $\mathscr{G}$ as an equational set, and verifying that the expression is universally valid on this set. This can be done algorithmically [5], but the algorithm is complicated and prohibitively resource-hungry even for simple families. Our algorithm, on the other hand, is not only very easy to implement, but also profits directly from any improvements to the subgraph isomorphism algorithm without the need to change a byte in our code.

As Head-Mid-Tail grammars are a special case of hyperedge replacement grammars, it follows that if an infinite family of graphs cannot be generated by hyperedge replacement grammars, then it cannot be generated by Head-Mid-Tail grammars. Because the number of edges in a hyperedge replacement grammar exhibits linear growth, hyperedge replacement families cannot contain cliques of arbitrary size. An example of graphs that contain arbitrary large cliques and therefore cannot be represented by HeadMid-Tail grammars are the anti-holes. All is not lost, however. Since $A$ is an induced subgraph of $B$ iff the complement of $A$ is an induced subgraph of the complement of $B$, and since holes can be represented by Head-Mid-Tail grammars, we can switch to the complement and still solve Problem 2. A prominent family where this trick does not work are the suns [8,7].

Another way to enhance the reach of our algorithm is taking unions of families. Fig. 8 shows the Dumbbells, which can be represented by a hyperedge replacement grammar, but not by a Head-Mid-Tail grammar. They cannot be represented by a finite union of Head-Mid-Tail grammars either. But when the cycles $x_{0}, \ldots, x_{i}$ and $z_{0}, \ldots, z_{k}$ are limited to length either 3 or 4 , we get the bicycles [3,7], which can be represented by a union of three Head-Mid-Tail grammars, with cycles of length 3 and 3; 3 and 4; and 4 and 4, respectively.

Considering the practical purpose of deciding relations between sets of forbidden subgraphs, we can say that surprisingly ${ }^{4}$ many families used in forbidden subgraph characterisations of existing graph classes are in fact representable by Head-Mid-Tail grammars. Of the roughly 100 infinite families currently used ${ }^{5}$ by ISGCI less than 10 cannot be represented by Head-Mid-Tail-Grammars and these cannot be represented by hyperedge replacement grammars, either. This implies that an improvement to our

\footnotetext{
${ }^{4}$ Actually not so very surprising, because Head-Mid-Tail grammars were developed with the goal to have a formalism that is as simple as possible and yet powerful enough to handle as many infinite families used by ISGCI as possible.

${ }^{5}$ Many more await being used; see Fig. 8 for some examples.
} 

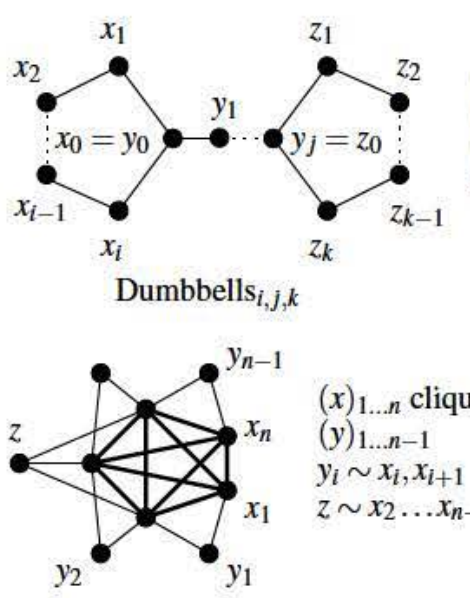

$(x)_{1 \ldots n}$ clique (bold)

(y) $)_{1 \ldots n-1}$

$y_{i} \sim x_{i}, x_{i+1}$

$z \sim x_{2} \ldots x_{n-1}$

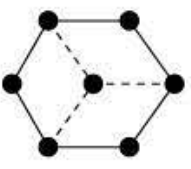

$(x)_{0 \ldots n}$ cycle

$z$ adjacent to at least one $x_{i}$ triangle-free

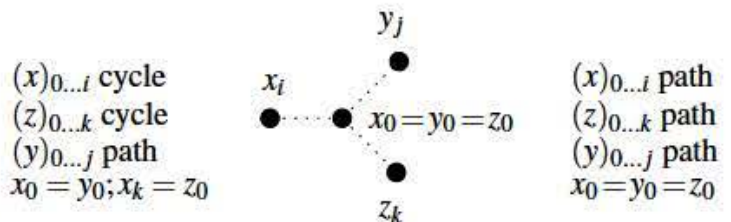

$\operatorname{Stars}_{i, j k}$

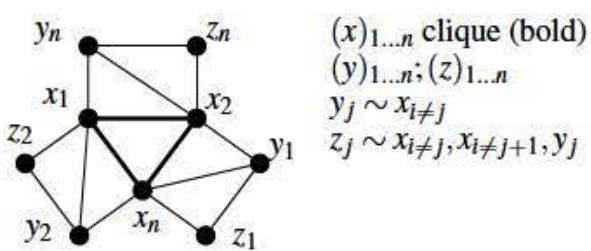

(no drawing)

$n, k$ relatively prime, $n>2 k$ $a_{i} \sim a_{j}$ with $j-i \leq k \bmod n$ $b_{i} \sim b_{j}$ with $j-i<k \bmod n$ $a_{i} \sim b_{j}$ with $j-i \leq k \bmod n$

Fig. 8. Typical examples of infinite families used in forbidden subgraph characterisations that cannot be generated by Head-Mid-Tail grammars. $(x)_{1 \ldots n}$ is shorthand for $x_{0} \ldots x_{n}$ and $x \sim y$ means $x$ ist adjacent to $y$.

algorithm should preferably act on a grammar more general than hyperedge replacement grammars.

Then there is the issue of deciding whether an infinite set $A$ forbids a finite or infinite set $B$. If $B$ is finite, Head-Mid-Tail grammars in $A$ work fine: Since only graphs that have at most as many vertices as the graphs in $B$ can forbid $B$, we can generate this finite set from the grammar and again check finite $A$ and $B$. But we currently cannot decide programmatically the case where both $A$ and $B$ are infinite.

This leaves us with the following open questions:

1. How far can Alg. 17 be extended easily? For example, a variation that results in derivation stars instead of a path seems straightforward and would already be powerful enough to represent dumbbells.

2. We think it is possible to extend the algorithm to work on hyperedge replacement grammars, but a naive extension would check an exponential number of graphs and therefore be impractical. What would a smarter extension look like?

3. In light of the families used by ISGCI, is there a practical algorithm for Problem 2 on grammars more powerful than hyperedge replacement, like pushout grammars?

4. The most burning open question of all is how to determine whether one Head-MidTail family forbids another one. 
5. When asking whether one grammar forbids another one, we expect to hit the undecidability barrier for powerful enough grammars. Is this correct and if so, where is this barrier?

\section{References}

1. Brandstädt, A., Le, V.B., Spinrad, J.P.: Graph Classes: A Survey. In: SIAM Monographs on Discrete Math. Appl., Philadelphia, vol. 3 (1999)

2. Chudnovsky, M., Robertson, N., Seymour, P.D., Thomas, R.: The strong perfect graph theorem. Annals of Mathematics 164, 51-229 (2006)

3. Chvátal, V., Hoàng, C.T., Mahadev, N.V.R., De Werra, D.: Four classes of perfectly orderable graphs. Journal of Graph Theory 11(4), 481-495 (1987)

4. Cordella, L., Foggia, P., Sansone, C., Vento, M.: A (sub)graph isomorphism algorithm for matching large graphs. IEEE Transactions on Pattern Analysis and Machine Intelligence 26(10), 1367-1372 (2004)

5. Courcelle, B., Engelfriet, J.: Graph structure and monadic second-order logic. Cambridge University Press (2012)

6. de Ridder, H.N., de Ridder, N.: The subgraph isomorphism problem on a class of hyperedge replacement languages. Technical report. Universität Konstanz, Fachbereich Informatik und Informationswissenschaft (2014)

7. de Ridder, H.N., et al.: Information System on Graph Classes and their Inclusions (ISGCI) (2001-2014), http://www.graphclasses.org

8. Farber, M.: Characterizations of strongly chordal graphs. Discrete Mathematics 43(23), 173-189 (1983)

9. Foggia, P., Sansone, C., Vento, M.: A performance comparison of five algorithms for graph isomorphism. In: Proceedings of the 3rd IAPR TC-15 Workshop on Graph-based Representations in Pattern Recognition, pp. 188-199 (2001)

10. Garey, M.R., Johnson, D.S.: Computers and Intractability: A Guide to the Theory of NPCompleteness. W. H. Freeman \& Co., New York (1979)

11. Habel, A.: Hyperedge Replacement: Grammars and Languages. LNCS, vol. 643. Springer, Heidelberg (1992)

12. König, D.: Theorie der endlichen und unendlichen Graphen, Leipzig (1936)

13. Lee, J., Han, W.-S., Kasperovics, R., Lee, J.-H.: An in-depth comparison of subgraph isomorphism algorithms in graph databases. In: Proceedings of the 39th International Conference on Very Large Data Bases, pp. 133-144 (2013)

14. Rozenberg, G. (ed.): Handbook of Graph Grammars and Computing by Graph Transformation. World Scientific Publishing Co., Inc. (1997)

15. Ullmann, J.R.: An algorithm for subgraph isomorphism. J. ACM 23(1), 31-42 (1976)

16. Vento, M., Foggia, P.: The vflib graph matching library, http://mivia.unisa.it/datasets/graph-database/vflib 